\title{
The temperature dependent structure of liquid 1-propanol as studied by neutron diffraction and EPSR simulations
}

\author{
Per Sillrén, ${ }^{1, \text { a) }}$ Jan Swenson, ${ }^{1}$ Johan Mattsson, ${ }^{2}$ Daniel Bowron, ${ }^{3}$ and Aleksandar Matic ${ }^{1}$ \\ ${ }^{1}$ Applied Physics, Chalmers University of Technology, SE-412 96 Göteborg, Sweden \\ ${ }^{2}$ School of Physics and Astronomy, University of Leeds, Leeds LS2 9JT, United Kingdom \\ ${ }^{3}$ ISIS Facility, Rutherford Appleton Laboratory, Chilton, Didcot, Oxon OX11 0QX, United Kingdom
}

(Received 9 March 2013; accepted 13 May 2013; published online 3 June 2013)

\begin{abstract}
The structure of liquid 1-propanol is investigated as a function of temperature using neutron diffraction together with Empirical Potential Structure Refinement modelling. The combined diffraction and computer modelling analysis demonstrates that propanol molecules form hydrogen bonded clusters with a relatively wide size distribution, which broadens at lower temperatures. We find that the cluster size distribution is well described by a recently proposed statistical model for branched H-bonded networks [P. Sillrén, J. Bielecki, J. Mattsson, L. Börjesson, and A. Matic, J. Chem. Phys. 136, 094514 (2012)]. The average cluster size increases from $\sim 3$ to 7 molecules, whilst the standard deviation of the size distribution increases from 3.3 to 8.5 as the temperature is decreased from 293 to $155 \mathrm{~K}$. The clusters are slightly branched, with a higher degree of branching towards lower temperatures. An analysis of the cluster gyration tensor $\left(R_{m n}\right)$ reveals an average elongated ellipsoidal shape with axes having proportions $1: 1.4: 1.9$. We find that the average radius of gyration has a cluster size dependence consistent with that of fractal clusters, $R_{g} \propto n^{1 / D}$, with a fractal dimension $D \approx 2.20$, which is close to $D=2.00$ expected for an ideal random walk or $D=2.11$ expected for reaction limited aggregation. The characteristic angles between the $\mathrm{H}$-bonded $\mathrm{OH}$-groups that constitute the clusters show only a weak temperature dependence with $\mathrm{O}-\mathrm{H}$. . O angles becoming more narrowly distributed around $180^{\circ}$ at lower temperatures. (C) 2013 AIP Publishing LLC. [http://dx.doi.org/10.1063/1.4807863]
\end{abstract}

\section{INTRODUCTION}

Hydrogen bonding has a strong influence on the properties of molecular liquids. One example is the large increase in the boiling and melting points of alcohols compared to their alkane analogs. Hydrogen bonding also contributes to complex thermodynamic behaviour, exemplified for instance by the anomalous properties of water. ${ }^{1-3}$ The hydrogen of an $\mathrm{OH}$-group can form a hydrogen bond (H-bond) with the oxygen of a neighbouring $\mathrm{OH}$-group. Each such hydrogen can participate in zero or one $\mathrm{H}$-bond and each oxygen has two lone electrons pairs that can participate in zero, one or two H-bonds. This leads to the formation of a network structure of OH-groups in hydrogen bonded liquids. The strength of a typical H-bond is $\sim 10 k_{B} T$ at room temperature, intermediate between that of the stronger covalent bond and the weaker van der Waals interaction, leading to a rapid $\left(10^{-12}-10^{-10} \mathrm{~s}\right)$ breaking and reforming of $\mathrm{H}$-bonds in the liquid state. ${ }^{4}$ The intermediate strength of $\mathrm{H}$-bonds also leads to a strong temperature dependence of $\mathrm{H}$-bond networks and thus also of the corresponding dynamic features related to the H-bond structure. Thus, a key to understand the properties of hydrogen bonding liquids is to describe the structure of the hydrogen bonded network and their temperature dependence.

While $\mathrm{H}$-bonds are playing important roles in a wide variety of materials based on complex chemistries, such as biological macromolecules or supramolecular systems, the much

\footnotetext{
a)Electronic mail: per.sillren@chalmers.se
}

simpler mono-hydroxy alcohols provide excellent model systems since the length of the alkyl part of the molecule and thus the H-bond density can be systematically controlled. The structure of alcohols has been investigated by experimental techniques such as X-ray and neutron diffraction, ${ }^{5-10}$ vibrational spectroscopy, ${ }^{11-13}$ and NMR. ${ }^{14,15}$ In fact, one of the first X-ray diffraction studies on liquids was performed on mono-hydroxy alcohols. ${ }^{16}$ An interesting feature found in $\mathrm{X}$ ray studies on mono-alcohols is the existence of a pre-peak in the structure factor, $F(Q)$, which has been suggested to be related to structural correlations in the H-bonded network. ${ }^{10,16}$ This peak is found to move to lower momentum transfer values with increasing number of carbons in the backbone of the molecule suggesting an increase in the correlation length within the H-bond network. Computer simulations are also often used to aid the analysis of diffraction data, providing real space configurations so that the $\mathrm{H}$-bonded structures can be analysed in detail. ${ }^{5-7,17-20}$

Despite the large interest and many performed studies no clear picture has yet emerged concerning the structure and topology of H-bonded networks in mono-hydroxy alcohols. The structures proposed in the literature range from ring structures, with a fixed number of 4-8 molecules in each cluster, ${ }^{21-25}$ to more elongated, sometimes branched, chaintype structures. $6,8,11,12,14,19,20,26-38$ The lack of a widely accepted structural model for mono-hydroxy alcohols clearly motivates further investigations.

To address this, we here study the mono-hydroxy alcohol 1-propanol, $\mathrm{C}_{3} \mathrm{H}_{7} \mathrm{OH}$, which has a back-bone 


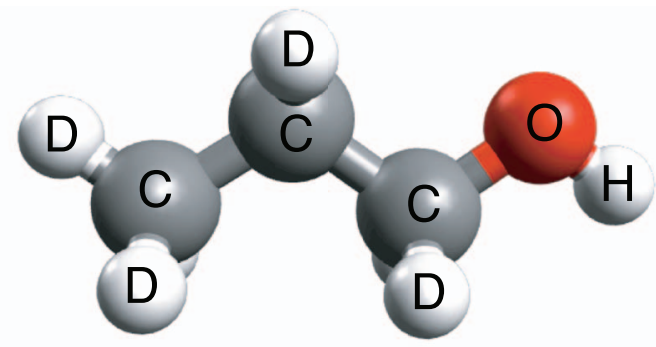

FIG. 1. A 1-propanol-D7 molecule with the carbons labeled C, the deuterium atoms labeled $\mathrm{D}$, the oxygen $\mathrm{O}$, and the hydrogen $\mathrm{H}$.

consisting of three carbon atoms together with one hydroxyl $(\mathrm{OH}-)$ group, as shown in Fig. 1. Previously, the structure of liquid 1-propanol has been investigated using X-ray and neutron diffraction, ${ }^{23,24}$ often combined with analysis techniques based on Reverse Monte Carlo (RMC), molecular dynamics, or Monte Carlo computer simulations. ${ }^{5,6}$ The H-bonded clusters are typically described as consisting of chain and/or ring structures with different size distributions. With the exception of one early X-ray study ${ }^{8}$ and a study of the glassy and crystalline states ${ }^{39}$ previous studies have all focused on the structure at ambient temperatures. In contrast, studies of the dynamics in monohydroxy alcohols are often performed over a wide temperature range to characterise the detailed behaviour of various relaxation processes. ${ }^{40-44}$ To properly correlate dynamics and structure, the temperature dependent structure has to be determined.

We here present results on the temperature dependence of the liquid structure, as obtained from neutron diffraction combined with EPSR (Empirical Potential Structure Refinement) simulations. The EPSR technique, which in contrast to RMC, uses a priori information about the molecular structure and interactions has been previously used together with diffraction data from other alcohols, such as methanol, ${ }^{28}$ tertiary butanol, ${ }^{7}$ and glycerol, ${ }^{18}$ as well as water. ${ }^{45}$ From our combined experimental and simulation study, we extract temperature dependent information on the topology, size, and shape of the H-bonded clusters. We quantify the cluster properties in terms of bonding probabilities, $\mathrm{H}$-bonding angles within the clusters, principal components of the gyration tensor of the clusters, and the average radius of gyration $\left\langle R_{g}\right\rangle$. We find that the cluster size distributions are well described by a recently proposed tree-model ${ }^{26}$ for hydrogen bonded clusters.

\section{EXPERIMENTAL AND COMPUTATIONAL METHODS}

\section{A. Neutron diffraction}

Neutron diffraction experiments were performed on 1propanol-D7 $\left(\mathrm{CD}_{3}-\mathrm{CD}_{2}-\mathrm{CD}_{2}-\mathrm{OH}\right)$ at 155,225 , and $293 \mathrm{~K}$, using the NIMROD diffractometer on the second target station of the ISIS spallation neutron source, UK. ${ }^{46}$ The outcome of the experiment is the total interference function, shown in Fig. 2, and given by

$$
F(Q)=\sum_{\alpha, \beta} c_{\alpha} c_{\beta} b_{\alpha} b_{\beta}^{*}\left(S_{\alpha, \beta}(Q)-1\right)
$$
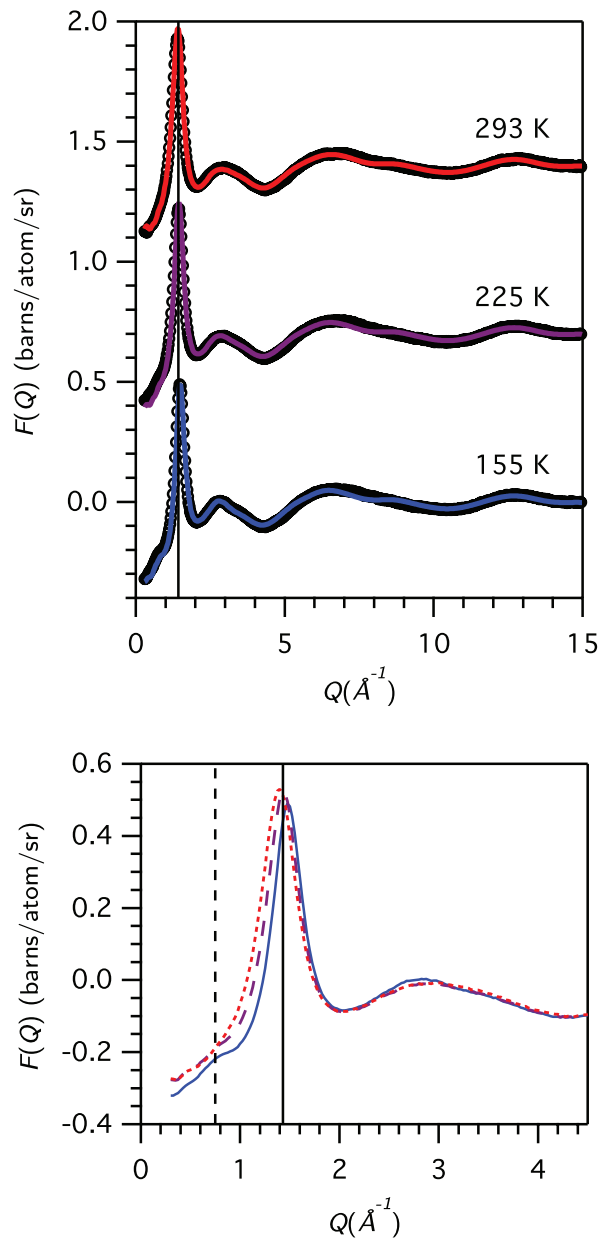

FIG. 2. (Top) Experimental total interference functions (circles) together with interference functions from the EPSR simulations (lines) at $155 \mathrm{~K}$, $225 \mathrm{~K}$, and $293 \mathrm{~K}$. The solid vertical line marks the position of the main peak at $225 \mathrm{~K}$. (Bottom) Experimental total interference functions plotted without a vertical shift for easier comparison between different temperatures: $155 \mathrm{~K}$ (solid blue), $225 \mathrm{~K}$ (dashed purple), and $293 \mathrm{~K}$ (dotted red). The solid vertical line marks the position of the main peak at $225 \mathrm{~K}$, whereas the dashed line indicates the position of the pre-peak.

where $c_{\alpha}$ is the concentration of atomic species $\alpha, b_{\alpha}$ is the neutron scattering length of species $\alpha$, and $S_{\alpha, \beta}(Q)$ is the partial structure factor of species $\alpha$ and $\beta$.

The sample was contained in a flat TiZr cell $(1 \mathrm{~mm}$ wall thickness and $1 \mathrm{~mm}$ sample thickness). The data were corrected for cell and instrument backgrounds, absorption and multiple scattering, and were normalised to absolute units using the scattering from a vanadium standard. The contribution from inelastic scattering, primarily due to hydrogen, was modelled as a short ranged single exponential, and subtracted from the total interference function, $F(Q)$. The data reduction and corrections were performed using the GudrunN software package (March 2012 version). ${ }^{47}$

1-Propanol-D7 (see Figure 1) was purchased from Cambridge Isotope Laboratories and used without further processing. The degree of deuteration was specified by the manufacturer to more than $98 \%$. 
TABLE I. Equilibrium bond lengths, bond angles, and Lennard-Jones parameters, masses, and Coulomb charges defining the reference potentials used in the simulations. $\mathrm{C}_{\mathrm{C}}$ is the central carbon atom. The values are taken from the OPLS-AA force field. ${ }^{49,50}$

\begin{tabular}{|c|c|c|c|c|}
\hline Bond & & & & Length $(\AA)$ \\
\hline$\overline{\mathrm{C}-\mathrm{C}}$ & & & & 1.529 \\
\hline C-D & & & & 1.090 \\
\hline $\mathrm{C}-\mathrm{O}$ & & & & 1.410 \\
\hline $\mathrm{O}-\mathrm{H}$ & & & & 0.945 \\
\hline Bond & & & & Angle \\
\hline$\overline{\mathrm{C}-\mathrm{C}-\mathrm{C}}$ & & & & $112.7^{\circ}$ \\
\hline $\mathrm{C}-\mathrm{C}-\mathrm{D}$ & & & & $110.7^{\circ}$ \\
\hline $\mathrm{C}-\mathrm{C}-\mathrm{O}$ & & & & $109.5^{\circ}$ \\
\hline D-C-D & & & & $107.8^{\circ}$ \\
\hline $\mathrm{D}-\mathrm{C}-\mathrm{O}$ & & & & $109.5^{\circ}$ \\
\hline $\mathrm{C}-\mathrm{O}-\mathrm{H}$ & & & & $108.5^{\circ}$ \\
\hline Atom type & $\varepsilon(\mathrm{kJ} / \mathrm{mol})$ & $\sigma(\AA)$ & $m$ (a.m.u.) & $q / e$ \\
\hline $\mathrm{C}$ & 0.276 & 3.50 & 12.0 & -0.180 \\
\hline $\mathrm{C}_{\mathrm{C}}$ & 0.276 & 3.50 & 12.0 & -0.120 \\
\hline $\mathrm{C}_{\mathrm{O}}$ & 0.276 & 3.50 & 12.0 & 0.145 \\
\hline $\mathrm{D}$ & 0.126 & 2.50 & 2.00 & 0.060 \\
\hline $\mathrm{O}$ & 0.711 & 3.12 & 16.0 & -0.683 \\
\hline $\mathrm{H}$ & 0 & 0 & 1.01 & 0.418 \\
\hline
\end{tabular}

\section{B. EPSR}

EPSR is a computational Monte Carlo method that minimises the difference between measured and simulated structure factors by iteratively refining the intermolecular potential used in the simulation. ${ }^{28}$ The EPSR method starts from physically plausible intra- and inter-molecular force fields, and tunes the intermolecular potential in order to minimise the difference between the calculated and experimental structure factors. The resulting structure is compatible with the measured structure factor and is physically sound from an energetic point of view.

For our simulations, we used 2100 molecules within a cubic box with periodic boundary conditions. The number densities were calculated by linear extrapolation between the densities of the two nearest temperatures, as reported in Ref. 48. For $155 \mathrm{~K}$, we used a density of $\rho=0.1104$ atoms $/ \AA^{-3}$, for $225 \mathrm{~K}$ we used $\rho=0.1032$ atoms $/ \AA^{-3}$, and for 293 we used $\rho=0.0967$ atoms $/ \AA^{-3}$. The intramolecular bond lengths and bond angles, as well as the reference intermolecular potential, were taken from the OPLS-AA force field, ${ }^{49,50}$ and are listed in Table I. Even though the intramolecular bonds are flexible, the average bond lengths and angles do not change significantly $(<2 \%)$ during the simulation.

\section{RESULTS AND DISCUSSION}

\section{A. Molecule conformations}

The molecular conformation of 1-propanol can be characterised by the equilibrium values of dihedral angles involving four atoms. The dihedral angle, $\phi$, between four atoms labeled 1-2-3-4, is illustrated in Figure 3. For the dihedral angles involving $\mathrm{C}-\mathrm{C}-\mathrm{C}-\mathrm{O}$ and $\mathrm{C}-\mathrm{C}-\mathrm{O}-\mathrm{H}$, there are five combinations, $\left(60^{\circ}, 60^{\circ}\right),\left(60^{\circ}, 180^{\circ}\right),\left(180^{\circ},-60^{\circ}\right),\left(180^{\circ}, 180^{\circ}\right)$,

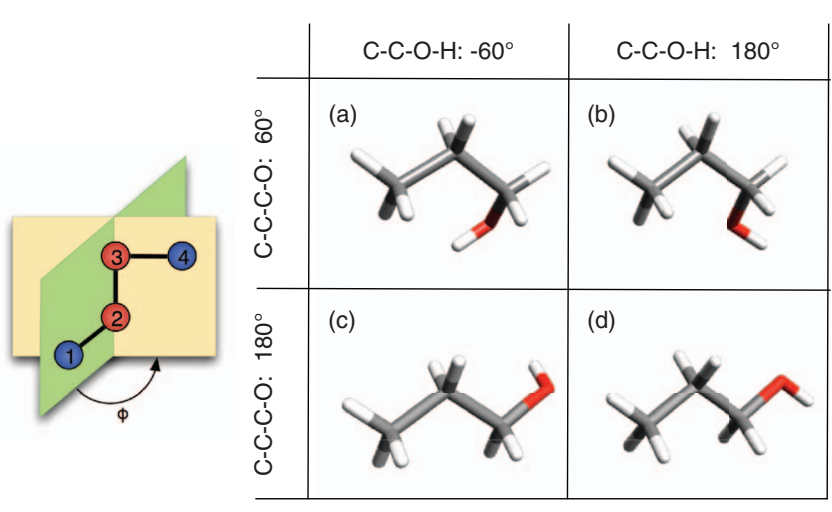

FIG. 3. (Left) The dihedral angle, $\phi$, between atoms 1-2-3-4 is the angle between the plane spanned by 1-2-3 and the plane spanned by 2-3-4. (Right) $\mathrm{C}-\mathrm{C}-\mathrm{C}-\mathrm{O}$ and $\mathrm{C}-\mathrm{C}-\mathrm{O}-\mathrm{H}$ dihedral angles used in the EPSR simulation.

and $\left(60^{\circ},-60^{\circ}\right)$ that have approximately the same intramolecular energy. ${ }^{49}$

To determine the optimal combination of conformations, we first simulated each of the five conformations separately to obtain the corresponding structure factors, $F_{a}=F_{60^{\circ},-60^{\circ}}(Q), \quad F_{b}=F_{60^{\circ}, 180^{\circ}}(Q), \quad F_{c}=F_{180^{\circ},-60^{\circ}}(Q)$, $F_{d}=F_{180^{\circ}, 180^{\circ}}(Q)$, and $F_{e}=F_{60^{\circ}, 60^{\circ}}(Q)$. Subsequently we minimised the difference (in a minimum square sense) between the measured structure factor, $F(Q)$ and the linear combination of the five simulated structure factors,

$$
\Delta=\int_{Q>1.75 \AA^{-1}}\left(F(Q)-a F_{a}-b F_{b}-c F_{c}-d F_{d}-e F_{e}\right)^{2} d Q,
$$

under the constraint $a+b+c+d+e=1$ for $Q>1.75 \AA^{-1}$, where the structure factors are dominated by intramolecular correlations. The best fit was obtained for $a=0.32, b=0.13$, $c=0.51, d=0.04$, and $e=0.00$. The three conformations $\mathrm{a}, \mathrm{b}$, and $\mathrm{c}$ have mirror conformations with dihedrals $(-60$, $60),(-60,180),(180,-60)$. Since these should occur with the same probability as their mirrored counterparts, the combination of conformations consists of 7 conformations with proportions $a_{ \pm}=0.16, b_{ \pm}=0.065, c_{ \pm}=0.255, d=0.04$.

The distribution of dihedral angles averaged over the production runs of the EPSR simulation is found in Figure 4. We note that the $\mathrm{C}-\mathrm{C}-\mathrm{C}-\mathrm{O}$ dihedral angle is largely unaffected by temperature. For the $\mathrm{C}-\mathrm{C}-\mathrm{O}-\mathrm{H}$ dihedral angle, on the other hand, angles between $80^{\circ}<|\phi|<160^{\circ}$ are more favoured at lower temperatures.

\section{B. Intermolecular structure}

Figure 2 shows the total interference functions $F(Q)$ obtained from experiments and simulations. For all three temperatures, we find a good agreement between the experiment and the simulation. The main peak in $F(Q)$ is found at $Q_{1}$ $=1.40 \AA^{-1}$ and distinct peaks can also be observed around 3,7 , and $13 \AA^{-1}$. The pre-peak, which is clearly seen in Xray diffraction experiments ${ }^{10}$ at $Q \approx 0.75 \AA^{-1}$, only shows up as a weak shoulder in the neutron data, see Figure 2, which becomes more pronounced for lower temperatures. The main peak is mainly related to intermolecular correlations and 

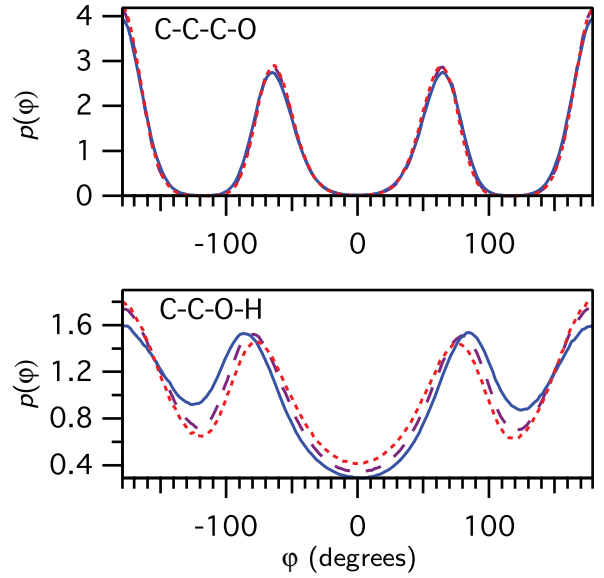

FIG. 4. (Top) $\mathrm{C}-\mathrm{C}-\mathrm{C}-\mathrm{O}$ dihedral angle distribution. (Bottom) $\mathrm{C}-\mathrm{C}-\mathrm{O}-\mathrm{H}$ dihedral angle distribution. Solid blue lines represent $155 \mathrm{~K}$, dashed purple lines $225 \mathrm{~K}$, and red dotted lines $293 \mathrm{~K}$.

shifts to slightly larger Q-values with decreasing temperature, $Q_{1}=1.43 \AA^{-1}$ at $225 \mathrm{~K}$ and $1.47 \AA^{-1}$ at $155 \mathrm{~K}$. The shift of the main peak can be attributed to the temperature induced density change discussed in Sec. II B. There is also a change in the region $2 \AA^{-1}<Q<4 \AA^{-1}$, corresponding to typical H-bonding distances, $1.57 \AA<2 \pi / Q<3.14 \AA$, with a small increase in the amplitude at lower temperatures.

From the EPSR simulations, we extract the partial structure factors and analyse their individual contributions to different regions in the interference function. In Figure 5, we show the intermolecular partial structure factors, obtained by subtracting the intramolecular contribution to the total partial structure factors, to highlight correlations between molecules. The main peak of $F(Q)$ clearly originates from the correlations between carbon atoms in one molecule and carbon, oxygen, and hydrogen atoms in other molecules. As shown in Figure 5, the largest temperature effects are found in structure factors involving $\mathrm{O}$ and $\mathrm{H}$ atoms. As these partials are directly related to the structure of the hydrogen bond network, it is clear that these structures change as a function of temperature. Also the pre-peak is strongly related to these partial structure factors (Figure 5, top frame), providing a strong link between the pre-peak and the characteristic length scales of the hydrogen bonded structures, discussed further in Sec. III C. The other partial structure factors have a negative contribution in this region. We thus conclude that
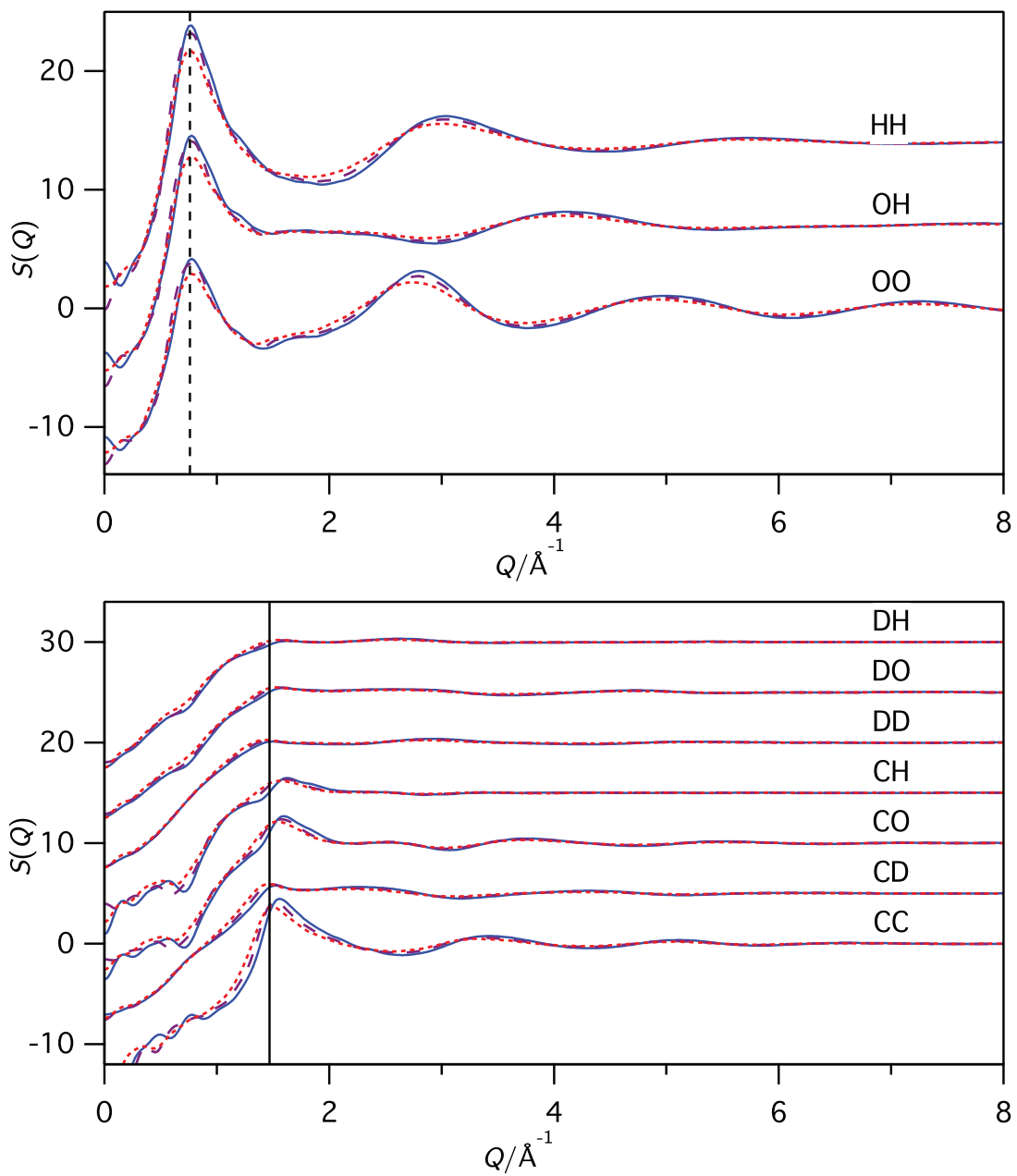

FIG. 5. Partial intermolecular structure factors, $S_{\alpha, \beta}^{\text {inter }}(Q)$ calculated from the EPSR simulations for the three temperatures $155 \mathrm{~K}$ (solid blue), $225 \mathrm{~K}$ (dashed purple), and $293 \mathrm{~K}$ (dotted red). The curves have been shifted vertically, with respect to each other, by 5 units in the lower frame and 7 units in the upper frame, for clarity. The dashed line in the upper frame highlights the contributions to the pre-peak in $F(Q)$, while the solid vertical line in the lower frame highlights the contributions to the main peak. 

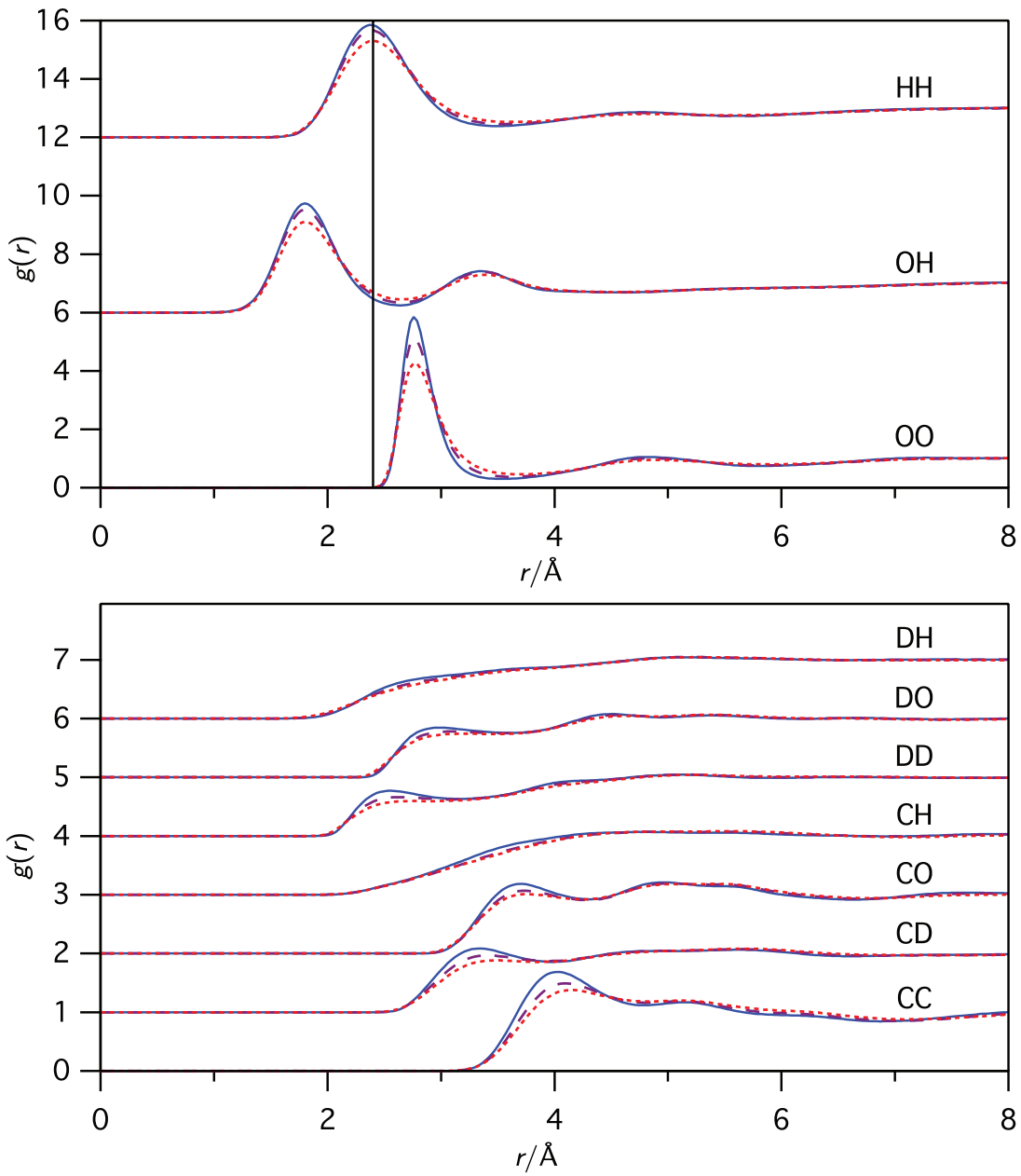

FIG. 6. Partial intermolecular radial distribution functions, $g_{\alpha, \beta}^{\text {inter }}(r)$ calculated from the EPSR simulations for the three temperatures $155 \mathrm{~K}$ (solid blue), $225 \mathrm{~K}$ (dashed purple), and $293 \mathrm{~K}$ (dotted red). The curves have been shifted vertically, by 1 unit in the lower frame and 6 units in the upper frame, for clarity. An H-bond is defined as two molecules having an intermolecular OH-distance less than 2.4 A. This distance is marked by a vertical line in the upper frame.

the absence of a distinct pre-peak in the neutron data is a consequence of the weighting of the partial structure factors in the neutron diffraction experiment, see Eq. (1). We also find that the partial structure factors involving the $\mathrm{O}$ and $\mathrm{H}$ atoms grow in intensity with decreasing temperature in the pre-peak region.

The partial intermolecular radial distribution functions $g_{\alpha, \beta}^{\text {inter }}(r)$, are shown in Figure 6. The typical $\mathrm{H} \cdot \mathrm{H}$ distance is found to be $2.4 \AA$, the typical $\mathrm{O}$...H distance $1.8 \AA$, and the typical O . . O distance $2.8 \AA$. With decreasing temperature the first peaks in the partial radial distribution functions become more well defined (sharper) even though the positions of the peaks do not change with temperature.

\section{Hydrogen bonding in liquid 1-propanol}

\section{Cluster sizes}

Using the obtained EPSR-configurations, hydrogen bonded structures in the liquid can be analysed and characterized in terms of size, shape, and topology. To define $\mathrm{H}-$ bonding, we use a simple maximum-distance criterion, where the distance between an oxygen atom and an H-bonded hydrogen is required to be less than $2.4 \AA$. The same distance criterion has previously been used to define hydrogen bonding in liquid methanol. ${ }^{28}$ It roughly represents the first minimum in the partial intermolecular pair distribution function $g_{\mathrm{OH}}^{\text {inter }}(r)$, as seen in Figure 6. The IUPAC definition of an Hbond $^{51}$ also contains an angle constraint on the H-bond: the $\mathrm{O}-\mathrm{H}-\mathrm{O}$ angle should preferably be above $110^{\circ}$. As can be seen in the top frame of Figure 10, our distance criterion results in a negligible fraction of $\mathrm{O}-\mathrm{H}-\mathrm{O}$ angles below $130^{\circ}$.

Figure 7 shows the cluster size distributions, as well as the weighted cluster size distributions determined from the EPSR configurations. While the cluster size distribution $r(n)$ gives the proportion of clusters of a specific size, the weighted cluster size distribution, $R(n) \propto n \times r(n)$, gives the proportion of molecules residing in clusters of a specific size. The average cluster size $\langle n\rangle$ is defined through

$$
\langle n\rangle=\sum_{n=1}^{\infty} n r(n),
$$

and the average weighted cluster size, i.e., the average cluster size a randomly chosen molecule belongs to, is defined 


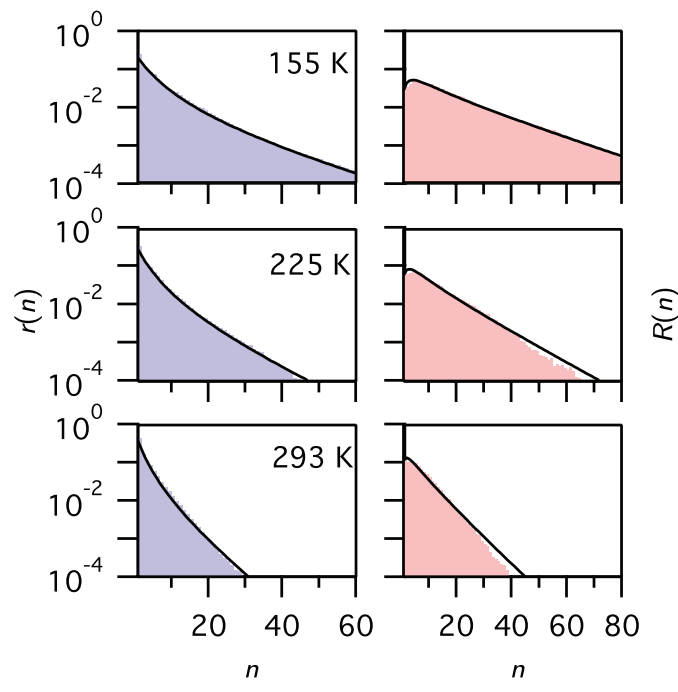

FIG. 7. (Left) Cluster size distributions, $r(n)$. (Right) Weighted cluster size distributions, $R(n)$. Solid lines show the distributions of the tree mode ${ }^{26}$ obtained by requiring them to have the same mean and variance as the cluster size distributions from the simulations, which are shown as bars.

through

$$
\langle n\rangle_{R}=\sum_{n=1}^{\infty} n R(n)=\frac{\sum_{n=1}^{\infty} n^{2} r(n)}{\sum_{n=1}^{\infty} n r(n)}
$$

We find that the average cluster size is fairly small, $\langle n\rangle=3.3$ at $293 \mathrm{~K}$. However, the distribution is rather wide, and we note that $90 \%$ of the molecules are in clusters containing less than 14 molecules. It is clear that with decreasing temperature the width of the distributions increases considerably. The average cluster sizes, $\langle n\rangle$ and $\langle n\rangle_{R}$, for the three temperatures are reported in Table II. We note that the average cluster sizes more than doubles when going from $293 \mathrm{~K}$ to $155 \mathrm{~K}$. At $225 \mathrm{~K}, 90 \%$ of the molecules are in clusters containing less than 24 monomers, and at $155 \mathrm{~K}, 90 \%$ are in clusters containing less than 39 monomers. The increased H-bonding at lower

TABLE II. Average cluster sizes, $\langle n\rangle$, average sizes of the cluster a randomly chosen $\mathrm{OH}$-group belongs to, $\langle n\rangle_{R}$, and bonding probabilities $p_{A}$ and $p_{B}$ obtained by requiring the model distributions to have the same mean and variance as the cluster size distributions from the simulations. The values of $p_{A}$ and $p_{B}$ calculated directly from the EPSR configurations are shown within parentheses, and were obtained by counting the fraction of oxygens with one $\left(f_{1}=p_{A}\left(1-p_{B}\right)\right)$ or two $\left(f_{2}=p_{A} p_{B}\right)$. H-bonds. $R_{g}$ is the clusters' average radius of gyration, $f_{\text {loop }}$ is the fraction of $\mathrm{OH}$-groups in closed loops, and $\langle n\rangle_{\text {loop }}$ is the average loop size. $\Delta H_{\text {vap }}$ denotes the heat of vaporisation.

\begin{tabular}{lccl}
\hline \hline & $155 \mathrm{~K}$ & $225 \mathrm{~K}$ & $293 \mathrm{~K}$ \\
\hline$\langle n\rangle$ & 6.88 & 4.73 & 3.21 \\
$\langle n\rangle_{R}$ & 17.37 & 10.8 & 6.54 \\
$p_{A}$ & $0.806(0.786)$ & $0.736(0.737)$ & $0.634(0.659)$ \\
$p_{B}$ & $0.060(0.095)$ & $0.072(0.080)$ & $0.085(0.064)$ \\
$\left\langle R_{g}\right\rangle$ & 4.48 & 3.80 & 3.23 \\
$f_{\text {loop }}$ & 0.08 & 0.06 & 0.05 \\
$\langle n\rangle_{\text {loop }}$ & 4.90 & 4.52 & 4.19 \\
$\Delta H_{\text {vap }}$ & 60.9 & 53.8 & 47.9 \\
\hline \hline
\end{tabular}

temperatures is also reflected in the increased heat of vaporisation, $\Delta H_{\text {vap }}$, also reported in Table II, which increases from 47.9 to 60.9 in going from 293 to $155 \mathrm{~K}$. The experimental value at $298 \mathrm{~K}$ is $47.3 \mathrm{~kJ} / \mathrm{mol}^{49}$

In Figure 7, we compare the results of the EPSRsimulations to our recently proposed statistical tree model for alcohols. ${ }^{26}$ This model uses two parameters to describe the two bonding sites on the oxygen; $p_{A}$ is the probability of forming a first bond between a given oxygen and a hydrogen atom on a neighbouring molecule and $p_{B}$ is the conditional probability of forming a second $\mathrm{H}$-bond, given that a first bond already exists. These probabilities are related to the first two moments of the distribution, $\langle n\rangle$ and $\left\langle n^{2}\right\rangle$, through, ${ }^{26}$

$$
\langle n\rangle=\frac{1}{1-p_{A}-p_{A} p_{B}}
$$

and

$$
\left\langle n^{2}\right\rangle=-\langle n\rangle+2\left(1-p_{A}\right)\langle n\rangle^{3} .
$$

Figure 7 shows the model distributions found by using Eqs. (4) and (5), requiring them to have the same mean and variance as the distributions from the simulations. Even though 5\%-8\% of the $\mathrm{OH}$-groups are found in closed loops, ${ }^{52}$ not accounted for by the tree model, the overall cluster size distributions are in good agreement with the model. The loops are either rings or parts of larger lasso-shaped clusters. By removing the $1 \%-2 \%$ of the $\mathrm{OH}$-bonds that are "closing the loops," all the clusters would have the topology described by the tree model.

The bonding probabilities can also be directly determined from the EPSR configurations by counting the fraction of oxygens with one $\left(f_{1}=p_{A}\left(1-p_{B}\right)\right)$ or two $\left(f_{2}=p_{A} p_{B}\right) \mathrm{H}-$ bonds. The bonding probabilities $p_{A}$ and $p_{B}$ thus obtained, together with the probabilities from fitting the tree model to the EPSR cluster size distribution are summarized in Table II. From these parameters, we conclude that the $\mathrm{OH}$-bonded clusters are not linear chains but are slightly branched, with $p_{B}$ ranging between 0.06 and 0.095 . We also note that the probability of obtaining a first hydrogen bond $p_{A}$ increases with decreasing temperature leading to a decrease in the fraction of monomers $\left(1-p_{A}\right)$ at lower temperatures. An increase of the branching probability $p_{B}$ with decreasing temperature is found from the EPSR configurations, but the opposite trend is obtained from fitting the tree model to the cluster size distribution. This discrepancy might be caused by the presence of lasso-shaped clusters in the EPSR configurations. Such lassoshaped clusters contribute to $f_{2}$, the fraction of oxygens $\mathrm{H}-$ bonded to two hydrogens without contributing to an increase of the average cluster size, otherwise implied by an increase in $f_{2}$. The tree model estimate of $p_{B}$ that is based on the average cluster size will thus be smaller than the real branching probability. However, we conclude that the overall agreement between the cluster size distributions obtained from the tree model and from the EPSR configurations provides support for the model's assumption of cluster size independent bonding probabilities. ${ }^{26}$ 


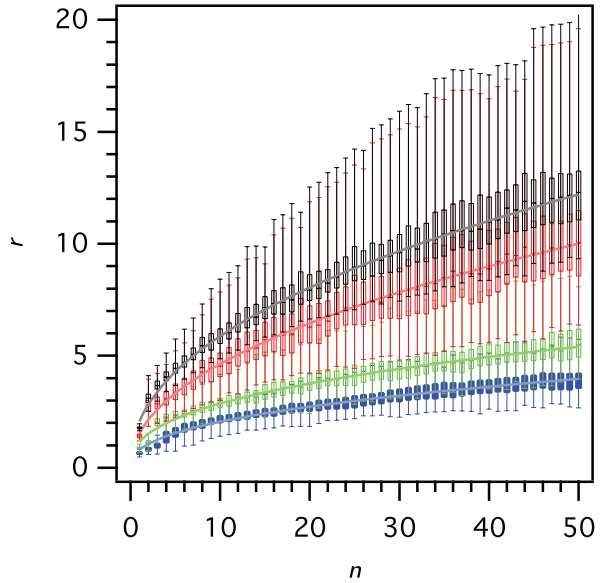

FIG. 8. Box plots of the principal components, $r_{1}$ (blue), $r_{2}$ (green), and $r_{3}$ (red), of the gyration tensor, $R_{n, m}$, as well as the radius of gyration $R_{g}$ (black), calculated from the H-bonded propanol clusters at $155 \mathrm{~K}$. Lines inside the boxes represent medians, box edges represent the 25th and 75th percentiles, and the whiskers show minimum and maximum values. The solid curves are power-law fits of the medians, see text for details.

The shape of the H-bonded clusters can be further analysed by calculating their gyration tensor

$$
R_{m n}=\frac{1}{N} \sum_{i=1}^{N} r_{m}^{(i)} r_{n}^{(i)}
$$

The eigenvalues $r_{1}, r_{2}$, and $r_{3}$, of the gyration tensor for the configuration at $155 \mathrm{~K}$ are plotted in Figure 8. The ratios of the principal components, $r_{1}: r_{2}: r_{3}$, obtained from fitting the individual components with power laws, $r_{i}=A n^{1 / D}$, is found to be 1:1.4:1.9. The fact that the ratios are different from one shows that the clusters are not spherical and need to be characterised by three different lengths. For clarity, only the principal components for $T=155 \mathrm{~K}$ are shown in Figure 8, but the principal components show very little temperature dependence for fixed cluster sizes, i.e., the only difference is that for lower temperatures, the calculated principal components extend to larger cluster sizes, $n$. Figure 8 also shows the radius of gyration, $R_{g}=\sqrt{r_{1}^{2}+r_{2}^{2}+r_{3}^{2}}$, as a function of cluster size. To extract the fractal dimension $D$ of the clusters, the radius of gyration medians have also been fitted with a power law, $R_{g}=A n^{1 / D}$, resulting in $A=2.06 \AA$, and a fractal dimension of, $D=2.20 \pm 0.02$. This value not far from $D=2$ expected for an ideal random walk and even closer to the value $\left(D \approx 2.11\right.$ ) found for reaction limited aggregation. ${ }^{53}$ Figure 9 shows the distribution of the radii of gyration, $R_{g}$, for the three investigated temperatures. The average radii of gyration, $\left\langle R_{g}\right\rangle$, are listed in Table II. The distributions have one sharp peak around $1.8 \AA$, which is related to monomers. The distributions also have a broader asymmetric peak, with average $5.4 \AA(155 \mathrm{~K}), 4.8 \AA(225 \mathrm{~K})$, and $4.3 \AA(293 \mathrm{~K})$. From $\left\langle R_{g}\right\rangle$ we note that the average cluster diameter, also corresponding to typical cluster-cluster distances, range between 6.5 and $9.0 \AA$ A It is interesting to note that this length-scale is similar to the one giving rise to the pre-peak in the interference function $F(Q)$. The pre-peak is found at $\sim 0.75 \AA^{-1}$ corresponding to real space distances of $\sim 2 \pi / 0.75=8.4 \AA$.

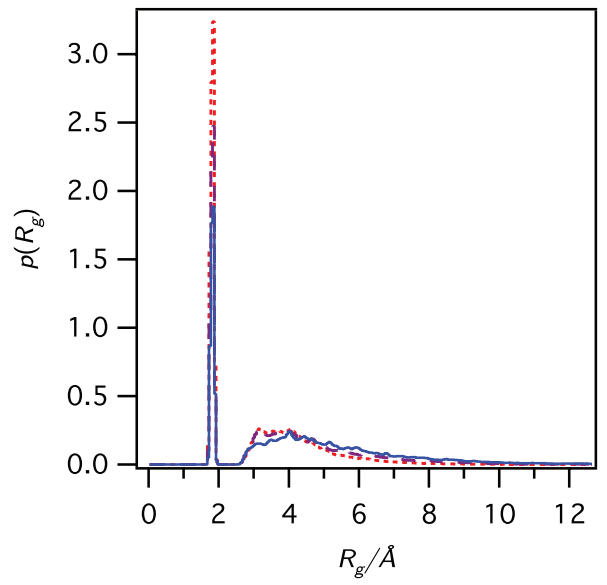

FIG. 9. Distribution of radii of gyration, $R_{g}$ for all $\mathrm{H}$-bonded clusters, and the temperatures $155 \mathrm{~K}$ (blue), $225 \mathrm{~K}$ (purple), and $293 \mathrm{~K}$ (red).

\section{Angles within the H-bonded clusters}

To further characterise the OH-bonded clusters, we have calculated the angles between two neighbouring $\mathrm{OH}$-groups, the $\mathrm{O}-\mathrm{H} \cdots \mathrm{O}$ and $\mathrm{H}-\mathrm{O} \cdots \mathrm{H}$ angles, where $\cdots$ represents a hydrogen bond, as well as the angles between three H-bonded $\mathrm{OH}$-groups, $\mathrm{O} \cdots \mathrm{O} \cdots \mathrm{O}$ and $\mathrm{H} \cdots \mathrm{H} \cdots \mathrm{H}$. The bond angle distributions from the simulated configurations are shown in Figure 10. The $\mathrm{H}-\mathrm{O} \cdots \mathrm{H}$ bond angle distribution is centred around $125^{\circ}$, which is the angle between a corner, the
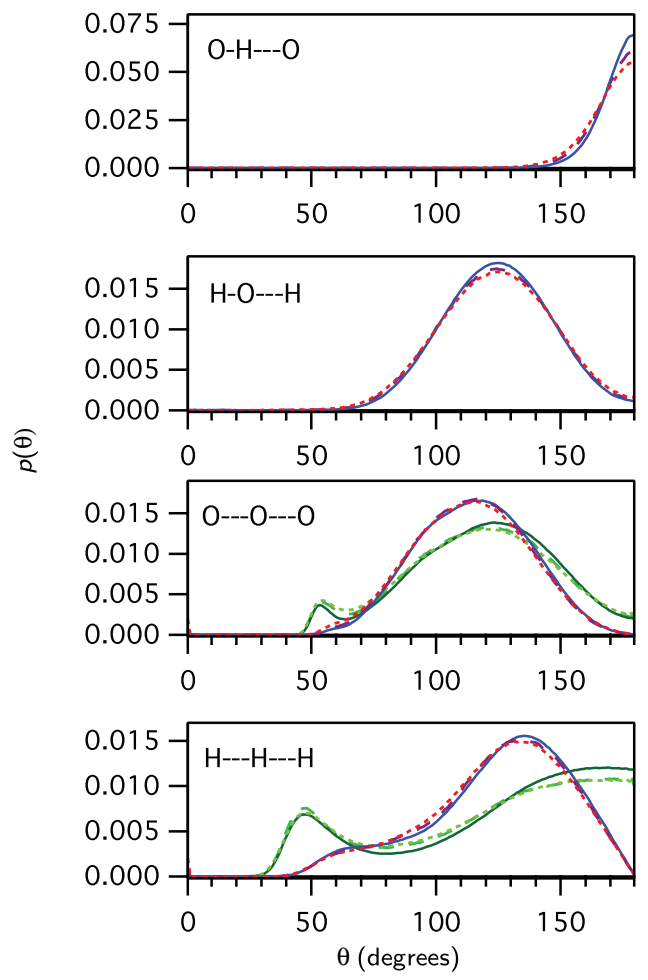

FIG. 10. Bond angle distributions for $\mathrm{O}-\mathrm{H} \cdots \mathrm{O}, \mathrm{H}-\mathrm{O} \cdots \mathrm{H}, \mathrm{O} \cdots \mathrm{O} \cdots \mathrm{O}$, and $\mathrm{H} \cdots \mathrm{H} \cdots \mathrm{H}$ triplets calculated for the different temperatures $155 \mathrm{~K}$ (solid blue), $225 \mathrm{~K}$ (dashed purple), and $293 \mathrm{~K}$ (dotted red). Blue, purple, and red curves show bond angle distributions for H-bonded triplets only, while the green curves also include some non-bonded triplets, see text for details. The situation is depicted in Figure 11. 


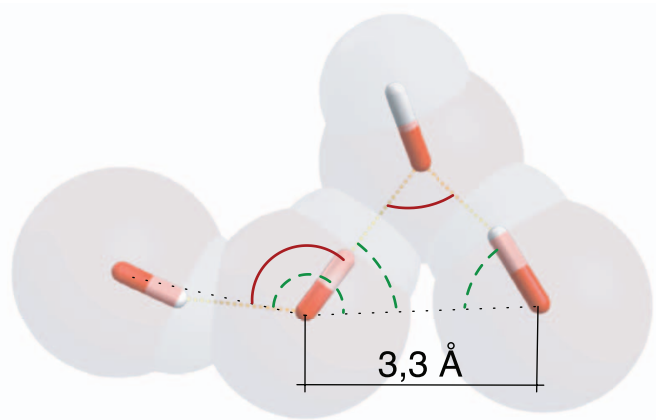

FIG. 11. Illustration of angles between neighbouring OH-groups. Only the two angles marked by red, solid arcs should be counted. Counting also the angles marked by green dashed arcs, results in a spurious peak at $54^{\circ}$, as well as too much weight to angles close to $180^{\circ}$ in the bond angle distribution, as can be seen in Figure 10.

centre, and the centre point of an edge in a tetrahedron. This means that the $\mathrm{H}$-bonded hydrogen is on average found right in between the two oxygen lone pairs. The $\mathrm{O} \cdots \mathrm{O} \cdots \mathrm{O}$ and the $\mathrm{H} \cdots \mathrm{H} \cdots \mathrm{H}$ bond angle distributions are shifted about $10^{\circ}$ down and up, respectively, and are centred around approximately $115^{\circ}$ and $135^{\circ}$. These distributions also have shoulders at approximately $60^{\circ}$ and $70^{\circ}$, respectively. The $\mathrm{O}-\mathrm{H} \cdots \mathrm{O}$ distribution, is centred around $180^{\circ}$, i.e., the hydrogen is on average located on a straight line between the two oxygens. The $\mathrm{O}-\mathrm{H} \cdots \mathrm{O}$, and the $\mathrm{H}-\mathrm{O} \cdots \mathrm{H}$ distributions show the strongest temperature dependence, both becoming narrower at lower temperatures.

For comparison with previously published bond angle distributions in glycerol and in water, ${ }^{54}$ Figure 10 also shows the bond angle distributions calculated by the "triangles" procedure directly available in the EPSR software. This uses a distance criteria $(2.0 \AA<d<3.4 \AA)$ for the $\mathrm{H} \cdots \mathrm{H}$ and O...O distances to decide whether the angles should be included in the distribution or not. However, using such a criterion, also angles of triplets that are not directly $\mathrm{H}$-bonded are included. The situation is illustrated with a sample cluster from the simulations in Figure 11, where the angles included in the EPSR bond angle distributions and not directly related to H-bonding are shown as green dashed arcs.

\section{CONCLUSIONS}

Neutron diffraction experiments on 1-propanol-D7 have been performed and analysed using EPSR simulations. We find that a mix of four different conformations of propanol, with different dihedral angles, is needed to correctly reproduce the measured interference function.

Our analysis of the configurations obtained from the EPSR simulations, based on the neutron diffraction data, supports the idea of branched chains, or tree-like, H-bonded clusters, with a rather broad size distribution. The average cluster size and the width of the cluster size distribution increases with decreasing temperature. We emphasise that according to our results, there is no "typical" cluster size that can be identified in the structure; in agreement with previous results on mono-alcohols ${ }^{5,17,19,20}$ the fraction of clusters of size $n$ decreases approximately exponentially with $n$. Nevertheless, average cluster sizes were calculated from the EPSR configurations and range from $\langle n\rangle=3.2$ at $293 \mathrm{~K}$, to $\langle n\rangle=6.8$ at $155 \mathrm{~K}$. The mean values of the weighted cluster size distributions, i.e., the cluster size a randomly chosen molecule resides in, range from $\langle n\rangle_{R}=6.5$ at $293 \mathrm{~K}$, to $\langle n\rangle_{R}=17$ at $155 \mathrm{~K}$. As the cluster sizes increase, with decreasing temperature, the fraction of non $\mathrm{H}$-bonded monomers decrease. We find that the cluster size distributions are well described by a recently proposed statistical model ${ }^{26}$ where the topology of the clusters is related to the bonding probabilities of the two oxygen lone-pairs. We note however, that a small fraction, $5-8 \%$ of the $\mathrm{OH}$-groups are in ring structures not accounted for by the model.

The results at $293 \mathrm{~K}$ are in good agreement with results by Vrhovsek et al. ${ }^{5}$ at $298 \mathrm{~K}$. For the cases where they include data from neutron- and X-ray diffraction, they obtain average cluster sizes in the range 3-6 molecules. Sahoo et al. ${ }^{23-25}$ compared room temperature neutron diffraction data of 1propanol to analytically calculated structure factors for four different scenarios: chains with 3, 4, or 5 molecules and rings with 6 molecules. They conclude that the hexametric rings best fit the data. As we have shown here, however, a mixture of (mostly chain-) clusters of different sizes can in fact reproduce the diffraction data quite well. Another study supporting ring structures, ${ }^{22}$ was based on comparing calculated thermodynamic properties, such as heat capacity and heat of vaporisation for chains, rings, and lasso-shaped clusters. The maximum cluster sizes considered were, however, not larger than 8. While the agreement between calculations and experimental data was the best for rings, the agreement improved for larger chain and lasso structures. From our EPSR simulations, we find that $27 \%$ of the molecules are in clusters of sizes larger than 8 , even at $293 \mathrm{~K}$, highlighting the need to include also larger cluster sizes when calculating thermodynamic properties.

The shape of the clusters, as determined by the principal components of the gyration tensor, can be described as ellipsoidal with the ratio of the principal axes approaching 1:1.4:1.9 for large cluster sizes $n$. The average radius of gyration, $R_{g}$, of the clusters approximately follows a behaviour with $R_{g} \propto n^{1 / 2.20}$. The radius of gyration distribution peaks at approximately $4 \AA$, corresponding to a centre-centre distance of $8 \AA$. An interesting observation is that the same length scale comes out from the position of the pre-peak in the partial structure factors involving $\mathrm{H}-\mathrm{H}, \mathrm{O}-\mathrm{H}$, and $\mathrm{O}-\mathrm{O}$ correlations.

Finally, the bond angle distributions within the $\mathrm{H}$ bonded clusters that are related to the approximately tetrahedral arrangement of covalently and $\mathrm{H}$-bonded atoms around the oxygen atoms show only a weak temperature dependence and slightly straighter $\mathrm{O}-\mathrm{H} \cdots \mathrm{O}$ angles at lower temperatures.

\section{ACKNOWLEDGMENTS}

We would like to acknowledge Alan Soper for helpful discussions regarding the data analysis and the Swedish Research Council for financial support.

${ }^{1}$ F. Franks, Water, A Comprehensive Treatise: The Physics and Physical Chemistry of Water (Plenum Press, New York, 1972), Vol. 1. 
${ }^{2}$ D. Eisenberg and W. Kauzmann, The Structure and Properties of Water (Oxford University Press, Oxford, 2005).

${ }^{3}$ H. E. Stanley, S. V. Buldyrev, M. Canpolat, M. Meyer, O. Mishima, M. R. Sadr-Lahijany, A. Scala, and F. W. Starr, Physica A 257, 213 (1998).

${ }^{4}$ E. Guàrdia, J. Marti, J. A. Padró, L. Saiz, and A. V. Komolkin, J. Mol. Liq. 96, 3 (2002).

${ }^{5}$ A. Vrhovsek, O. Gereben, A. Jamnik, and L. Pusztai, J. Phys. Chem. B 115, 13473 (2011).

${ }^{6}$ I. Akiyama, M. Ogawa, K. Takase, T. Takamuku, T. Yamaguchi, and N. Ohtori, J. Solution Chem. 33, 797 (2004).

${ }^{7}$ D. Bowron, J. Finney, and A. Soper, Mol. Phys. 93, 531 (1998).

${ }^{8}$ A. Mikusinska-Planner, Acta Cryst. A 33, 433 (1977).

${ }^{9}$ I. Bakó, P. Jedlovszky, and G. Pálinkás, J. Mol. Liq. 87, 243 (2000).

${ }^{10}$ M. Tomšič, A. Jamnik, G. Fritz-Popovski, O. Glatter, and L. Vlček, J. Phys. Chem. B 111, 1738 (2007).

${ }^{11}$ F. Palombo, P. Sassi, M. Paolantoni, and A. Morresi, J. Phys. Chem. B 110, 18017 (2006).

${ }^{12}$ M. Paolantoni, P. Sassi, A. Morresi, and R. Cataliotti, J. Raman Spectrosc. 37, 528 (2006).

${ }^{13}$ K. Lin, X. Zhou, Y. Luo, and S. Liu, J. Phys. Chem. B 114, 3567 (2010).

${ }^{14}$ C. Gainaru, R. Meier, S. Schildmann, C. Lederle, W. Hiller, E. Rössler, and R. Böhmer, Phys. Rev. Lett. 105, 258303 (2010).

${ }^{15}$ M. Poeschl and H. G. Hertz, J. Phys. Chem. 98, 8195 (1994).

${ }^{16}$ G. W. Stewart and R. M. Morrow, Phys. Rev. 30, 232 (1927).

${ }^{17}$ T. Yamaguchi, K. Hidaka, and A. Soper, Mol. Phys. 96, 1159 (1999).

${ }^{18}$ J. Towey, A. Soper, and L. Dougan, Phys. Chem. Chem. Phys. 13, 9397 (2011).

${ }^{19}$ P. Gómez-Álvarez, L. Romaní, and D. González-Salgado, J. Chem. Phys. 138, 044509 (2013).

${ }^{20}$ J. Lehtola, M. Hakala, and K. Hämäläinen, J. Phys. Chem. B 114, 6426 (2010).

${ }^{21}$ S. W. Benson, J. Am. Chem. Soc. 118, 10645 (1996).

${ }^{22}$ R. Ludwig, Chem. Phys. Chem. 6, 1369 (2005).

${ }^{23}$ A. Sahoo, S. Sarkar, P. S. R. Krishna, V. Bhagat, and R. N. Joarder, Pramana, J. Phys. 71, 133 (2008).

${ }^{24}$ A. Sahoo, S. Sarkar, V. Bhagat, and R. N. Joarder, J. Phys. Chem. A 113, 5160 (2009).

${ }^{25}$ A. Sahoo, S. Sarkar, P. Krishna, and R. N. Joarder, Pramana, J. Phys. 74, 765 (2010).

${ }^{26}$ P. Sillrén, J. Bielecki, J. Mattsson, L. Börjesson, and A. Matic, J. Chem. Phys. 136, 094514 (2012).

${ }^{27}$ M. Paolantoni, P. Sassi, A. Morresi, and R. Cataliotti, Chem. Phys. 310, 169 (2005).
${ }^{28}$ A. Soper, Chem. Phys. 202, 295 (1996).

${ }^{29}$ E. Tsuchida, Y. Kanada, and M. Tsukada, Chem. Phys. Lett. 311, 236 (1999).

${ }^{30} \mathrm{H}$. Torii, Pure Appl. Chem. 76, 247 (2004).

${ }^{31}$ T. Kosztolanyi, I. Bakó, and G. Pálinkás, J. Chem. Phys. 118, 4546 (2003).

${ }^{32}$ K. Vahvaselka, R. Serimaa, and M. Torkkeli, J. Appl. Crystallogr. 28, 189 (1995).

${ }^{33}$ H. Graener, T. Q. Ye, and A. Laubereau, J. Chem. Phys. 90, 3413 (1989).

${ }^{34}$ H. Graener, T. Q. Ye, and A. Laubereau, J. Chem. Phys. 91, 1043 (1989).

${ }^{35}$ L. Saiz, J. A. Padró, and E. Guàrdia, J. Phys. Chem. B 101, 78 (1997).

${ }^{36}$ J. A. Padró, L. Saiz, and E. Guàrdia, J. Mol. Struct. 416, 243 (1997).

${ }^{37}$ M. Haughney, M. Ferrario, and I. McDonald, J. Phys. Chem. 91, 4934 (1987).

${ }^{38}$ I. Svishchev and P. Kusalik, J. Chem. Phys. 100, 5165 (1994).

${ }^{39}$ G. J. Cuello, C. Talón, F. J. Bermejo, and C. Cabrillo, Appl. Phys. A 74, s552 (2002).

${ }^{40}$ C. M. Roland, R. Casalini, R. Bergman, and J. Mattsson, Phys. Rev. B 77, 012201 (2008).

${ }^{41}$ L. Wang, C. Angell, and R. Richert, J. Chem. Phys. 125, 074505 (2006).

${ }^{42}$ L. Wang and R. Richert, J. Phys. Chem. B 109, 8767 (2005).

${ }^{43}$ O. Kalinovskaya and J. Vij, J. Chem. Phys. 112, 3262 (2000).

${ }^{44}$ C. Hansen, F. Stickel, T. Berger, R. Richert, and E. Fischer, J. Chem. Phys. 107, 1086 (1997).

${ }^{45}$ A. Soper, J. Phys. Condens. Matter 19, 335206 (2007).

${ }^{46}$ D. T. Bowron, A. K. Soper, K. Jones, S. Ansell, S. Birch, J. Norris, L. Perrott, D. Riedel, N. J. Rhodes, and S. R. Wakefield, Rev. Sci. Instrum. 81, 033905 (2010)

${ }^{47}$ A. Soper, Rutherford Appleton Laboratory Technical Reports RAL-TR2011-013 (2011).

${ }^{48}$ Z. R. Zakaryaev, J. Eng. Phys. 43, 1261 (1982).

${ }^{49}$ W. L. Jorgensen, D. S. Maxwell, and J. TiradoRives, J. Am. Chem. Soc. 118, 11225 (1996)

${ }^{50}$ W. Damm, A. Frontera, J. Tirado-Rives, and W. L. Jorgensen, J. Comput. Chem. 18, 1955 (1997).

${ }^{51}$ E. Arunan, G. R. Desiraju, R. A. Klein, J. Sadlej, S. Scheiner, I. Alkorta, D. C. Clary, R. H. Crabtree, J. J. Dannenberg, P. Hobza, H. G. Kjaergaard, A. C. Legon, B. Mennucci, and D. J. Nesbitt, Pure Appl. Chem. 83, 1637 (2011).

${ }^{52}$ The fraction of molecules in loops were determined from the simulations by using the "rings" routine in the EPSR software.

${ }^{53}$ W. D. Brown and R. C. Ball, J. Phys. A 18, L517 (1985).

${ }^{54}$ L. Dougan, S. Bates, R. Hargreaves, J. Fox, J. Crain, J. Finney, V. Reat, and A. Soper, J. Chem. Phys. 121, 6456 (2004). 